\title{
A Note on Hebosteotomy.*
}

\author{
By A. J. Wallace, M.D., \\ Surgeon to the Hospital for Women, and to the Maternity Hospital, \\ Liverpool.
}

Six years ago (1906) the writer reported two cases of hebosteotomy by the open method (Trans. North of Eng. Obstet. and Gynæcol. Soc., 1906 , p. 117). The results of these were by no means such as to encourage repetition of the operation, for the liability to hæmorrhage, the possibility of laceration of hollow viscera and the difficulty of keeping the wound clean subsequently, seemed excellent reasons for preferring Casarean section. Nevertheless I felt that in suitable cases an improved technique should render the procedure one of value, and it seemed to me, as the result of the two experiences mentioned, that a suitable case was one in which the pelvic contraction was of moderate degree, and where the addition of another centimetre to the conjugata vera offered with tolerable certainty the delivery of a living child.

The unfarourable results reported year by year from Continental clinics deterred me from making use of the operation even in cases in which its practicability was entertained. There was one exception to this, a case in which the attempt to pass Döderlein's sawcarrying needle failed because the latter was too short to reach the lower border of an unusually deep pubic bone.

In the course of the present year two cases presented themselves in which the necessary conditions for the operation existed, i.e., the degree of pelvic contraction was moderate and yet forcible extraction by forceps implied such pressure on the foetal head that the birth of a dead child appeared inevitable. The cases are as follows:-

CASE I.-Mrs. E. L., aged 25; a primipara at full term : admitted to the Maternity Hospital, March 10 1912. During the pregnancy there had been vomiting daily, and latterly vulvar œdema and albuminuria had been noted.

When called to see the case, the medical attendant recognized a degree of pelvic contraction which seemed to contraindicate delivery per vias naturales, and sent the patient by ambulance into hospital.

The pelvis was found to be a generally contracted one with a Cv. of 3 inches. Under anæsthesia, manual exploration ascertained :

the cervix fully dilated: I9I2.

* Based on a paper read before the British Medical Institution, Oct. 24 
an enormous caput covering that portion of the head which projected into the pelvis:

the head lying in the R.O.P. position, with the occiput presenting.

The head was pushed out of the pelvis and rotated into the R.O.A. position, and forceps applied, but without effecting any advance.

In view of the general pelvic contraction, greater than usual at the outlet where there was an immobile coccyx, it was clear that a living child could not be delivered unless the pelvis were enlarged or Cæsarean section performed.

Hebosteotomy was done at once, the divided ends of the bones gaping to the extent of $\frac{3}{4}$ inch. Vaginal examination showed that no damage had been inflicted on the soft parts, so the patient was placed in the lithotomy position, each leg being held by a nurse who also exerted slight pressure against her side of the patient's pelvis. The forceps was applied and the head was easily drawn down into the pelvis, the gap increasing to a full 2 inches. The vulvar opening was small and a right episiotomy was done to preserve the perinæum.

A living female child weighing 7 lbs. was delivered.

Very little bleeding occurred from the small skin incisions for the passage of carrier and saw, nor was there any indication of bleeding in the deeper parts of the wound. Bladder, urethra and vagina were intact.

Convalescence was delayed only by soreness of the skin of the left labium majus, due to the too vigorous application of preparatory lotion prior to operation. This confined the patient to bed for seventeen days and was accountable for some slight irregularity of temperature. She was out of bed on the seventeenth day, and two days later walked about in comfort. She left hospital on the 24th day and spent the first five weeks at home resting, getting about gradually. At the end of that time she was perfectly well, and able to perform all her domestic duties.

She was examined a week or two ago when the pelvis was $x$-rayed. On palpation, there appeared to be a gap about rather less than one centimetre wide between the ends of the bones. The external osseous edge was sharply defined, the inner more rounded and less firm. The pelvic girdle was quite rigid and this rigidity did not appear to be affected by the patient's movements. The radiogram of this patient's pelvis shows a condition which is at least unusual. There is an apparent gap of great width between the pubic bones, but close inspection of this gap shows that it is formed of three distinct segments :-(i) in the middle line, the fibro-cartilage of the symphysis pubis; (ii) on the left side of this should appear the inner fragment of the divided left os pubis, but in the radiogram it is practically invisible except for some faint granular markings adja- 


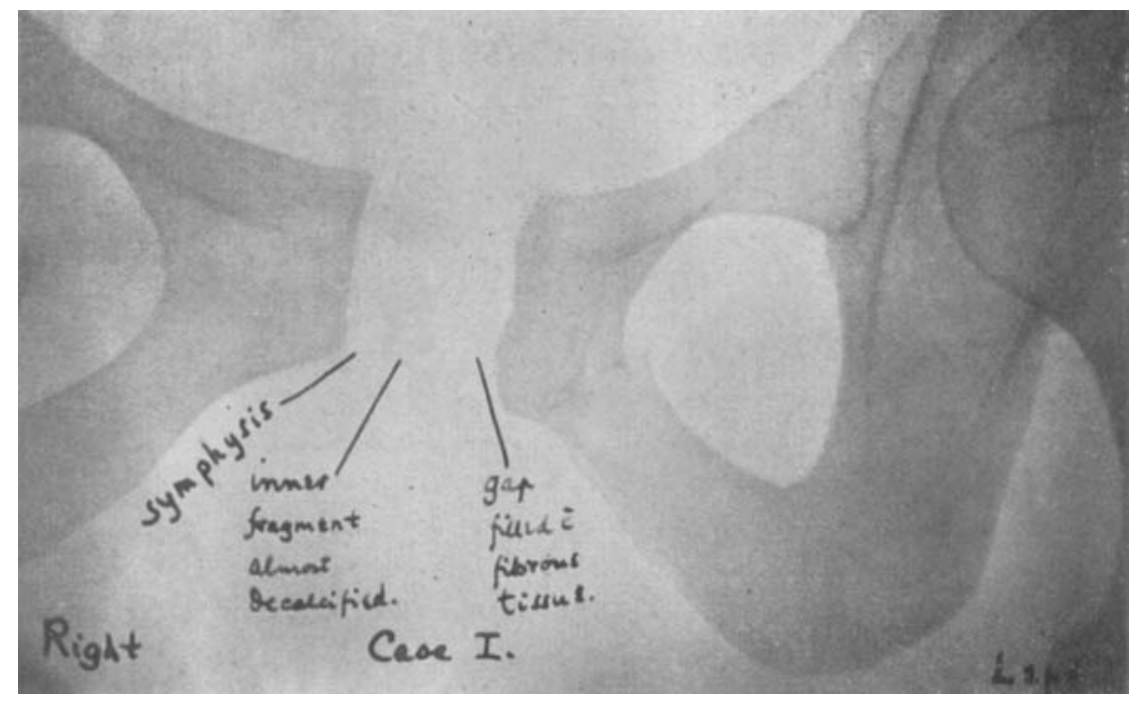

CAsE I.--Patient lying on her face.

Syuphysis. Osteotomy.

।

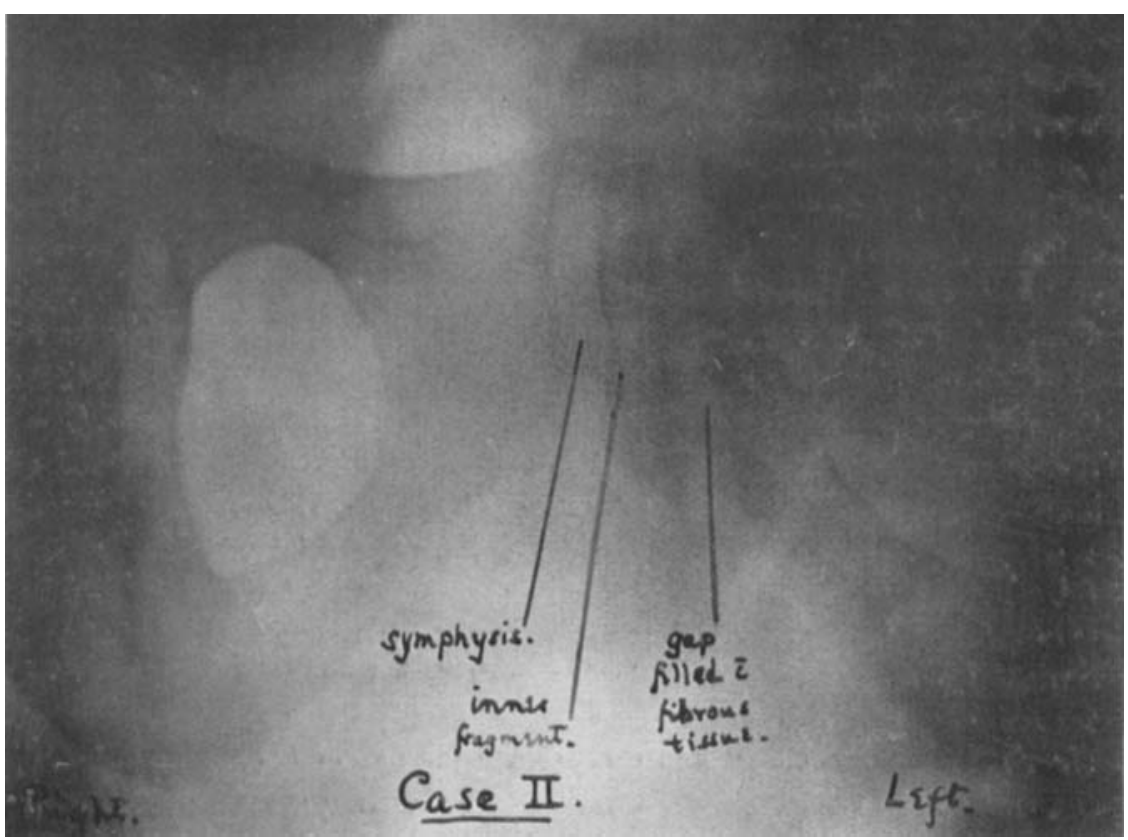

Case II.-Lying face down. 
cent to the upper part of the symphysis. Careful inspection enables one to distinguish a faint difference between the area of the invisible inner fragment and (iii) the gap between it and the external fragment of the os pubis. Mr. C. Thurstan Holland, who kindly took the radiogram, considers that the lime salts have been absorbed from the inner fragment. This condition must be unusual, as I have been unable to discover any mention of it. The explanation in all probability will be found in some alteration of the nutritive supply to the inner fragment.

CASE II.-Mrs. MoC., aged 40 : multipara at full term : admitted to the Maternity Hospital Sept. 6 1912. The pregnancy had been uneventful. There had been ten full term deliveries and one abortion. The 1st, 2nd, 4th and 5th labours were terminated naturally: the others were forceps deliveries. Five of the children had died within the first month from, so far as could be gathered, injuries received during labour.

Labour began at 2 a.m. on September 6, the membranes rupturing about the same time. During the day the midwife in charge of the case summoned Dr. H. R. MacIntyre, and he recognized that labour was obstructed and sent the patient into hospital. A well-marked retraction ring was found at the umbilical level, the cervix was fully dilated and the head lay transversely across the pelvic brim with the occiput pointing to the mother's right side. A prominent sacral promontory jutted into a deep dinge in the posterior parietal bone, whilst the right side of the head bulged forwards above the symphysis. The pelvis was a flat one without general contraction, the Cv. being estimated at $3 \frac{1}{2}$ inches. The child was alive.

Hebosteotomy was performed through the left pubic bone, and forceps was at once applied. The head came through the pelvis with the greatest ease, the divided bones separating to the extent of $2 \frac{1}{2}$ inches. A living male child weighing 8 lbs. $1 \mathrm{oz}$. was delivered. Very little hæmorrhage occurred from the operation nicks through the skin, and the hollow viscera were intact.

Convalescence was normal. On the 5th day some thrombosis was discovered in a few superficial varicose veins of the legs, but there was no constitutional disturbance. The patient was able to move about in bed from the first: she got up on the 12th day and left hospital on the 15th, quite able to walk and stand. She was seen last when $x$-rayed and was able to get about in the ordinary way.

The child was cyanosed when born and artificial respiration was required to resuscitate it. The head was asymmetrical, the flattened (posterior) right parietal bone showed a dint $\frac{1}{2}$ inch in depth, and there was also a shallower dint on the left parietal, presumably where it had been driven against a pubic bone. A large cephal- 
hæmatoma subsequently developed over the right parietal bone. Six weeks after delivery a radiogram of the pelvis was taken. It shewed a well marked gap between the two fragments of the os pubis, and an inner fragment with practically parallel sides, and presenting the usual appearance of normal bone.

At this time the pelvic girdle was quite rigid, and the patient was able to go about in the usual way and make all the ordinary movements without discomfort.

With regard to the performance of the operation, the method adopted was identical in each case. Döderlein's saw carrier was passed through a small nick in the skin midway between symphysis and pubic spine: its point was pushed through the fascial structures of the abdominal wall, and with the help of a finger passed per vaginam, guided along the posterior aspect of the left pubic bone and then forwards round its lower border, when it was made to project against the skin immediately external to the left labium majus. Here a nick was made sufficient to allow the point to protrude for the attachment of a Gigli's wire saw. Exact orientation is not easy especially if the patient be fat, but my endeavour was to keep the saw parallel to the symphysis.

In most diagrams illustrating the performance of the operation the patient is shewn in the lithotomy position, which is undoubtedly convenient both for the operation and the subsequent delivery. But I think the lithotomy position is unsuitable for the actual hebosteotomy because in that position the great adductor muscles of the thighs and the pubo-femoral ligaments of the hip-joints are tensely stretched, with the result that as soon as the bones are divided the sawn surfaces suddenly spring asunder with some violence. As a result serious lacerations of the soft parts may occur quite apart from injuries produced by the passage of the child. Therefore, in the two cases related, hebosteotomy was performed whilst the patient was in the recumbent position with the thighs slightly abducted. After division of the bone the lithotomy position was adopted, a nurse being in charge of each lower limb and also making slight pressure against the great trochanter of her own side. With these precautions it was found that the primary gap was less marked than in cases in which the lithotomy position was used throughout. Probably the use of the Walcher position for hebosteotomy would be still more advantageous and I shall certainly give it a trial in the future.

In both cases immediate extraction with forceps was carried out. In a case that is suitable for the operation I cannot perceive any gain in leaving delivery to the natural powers. To follow the latter course implies with every pain disturbance of the operation field and intermittent compression of injured blood vessels from which fresh 
bleeding may occur: therefore I think it is better to apply forceps and at once end the labour.

The Position of Hebosteotomy amongst other Obstetric Operations.

The operation is comparatively new, and in recent years Continental obstetricians have performed it with more zeal than discretion. The statistics obtained at the Dresden klinik may be given as an example of the unfortunate results of its wholesale performance :

(Roth. Centralb. f. Gyn., 1911, p. 362).

83 women were operated upon.

In 85 operations there were 2 maternal deaths $=2 \cdot 35$ per cent. mortality, and 6 children died $=7$ per cent. mortality.

Complications during Operation.

Hamorrhage severe in 13 cases; very severe in 3 cases. Checked in all by compression.

Collapse occurred in 1 case, thought to be due to internal bleeding. The patient recovered very slowly.

Injuries to soft parts.

Vagina: in 10 primiparæ lacerations communicating with the sawn ends of the bone, 19.4 per cent.

In 5 multiparæ lacerations communicating with the sawn ends of the bone, $9 \cdot 8$ per cent.

In 1 case the laceration of the vagina occurred immediately after division of the bone and before delivery was attempted.

Bladder : in 6 cases of severe vaginal lacerations the bladder was also torn.

In 6 cases the bladder only was lacerated.

Sacro-iliac Joints: "slight lesions" of the ligaments were found in isolated instances. In 1 fatal case bilateral suppurative arthritis was found at the necropsy.

In the Puerperium.

The morbidity was raised (the standard is not stated) : 48 cases came under this heading $=56.5$ per cent. There was severe fever in 20 cases ( 23.5 per cent.) and thrombophlebitis occurred in 14 per cent.

Subsequent investigation into 38 patients (40 operations).

Ten complained of difficulty in locomotion and in working.

Nine complained of pelvic pain due to lesions of the sacro-iliac joints.

In all these 19 the disproportion between head and pelvis had been very marked. 
21 had pronounced weakness of the pelvic floor.

In 20 cases subsequent labours were observed.

In 6 cases the subsequent labour was favourably influenced.

In the remainder bony union of the divided bones had occurred so that no advantage accrued in the second labour.

As the results of these experiences hebosteotomy had been excluded as the operation of choice in all primiparæ, i.e., in the Dresden Klinik. Cæsarean section is chosen for such cases. Hebosteotomy is regarded as unsuitable for performance in private houses.

Schläfli and Roemer have between them collected 1,000 cases, and the results are tabulated as follows:- 


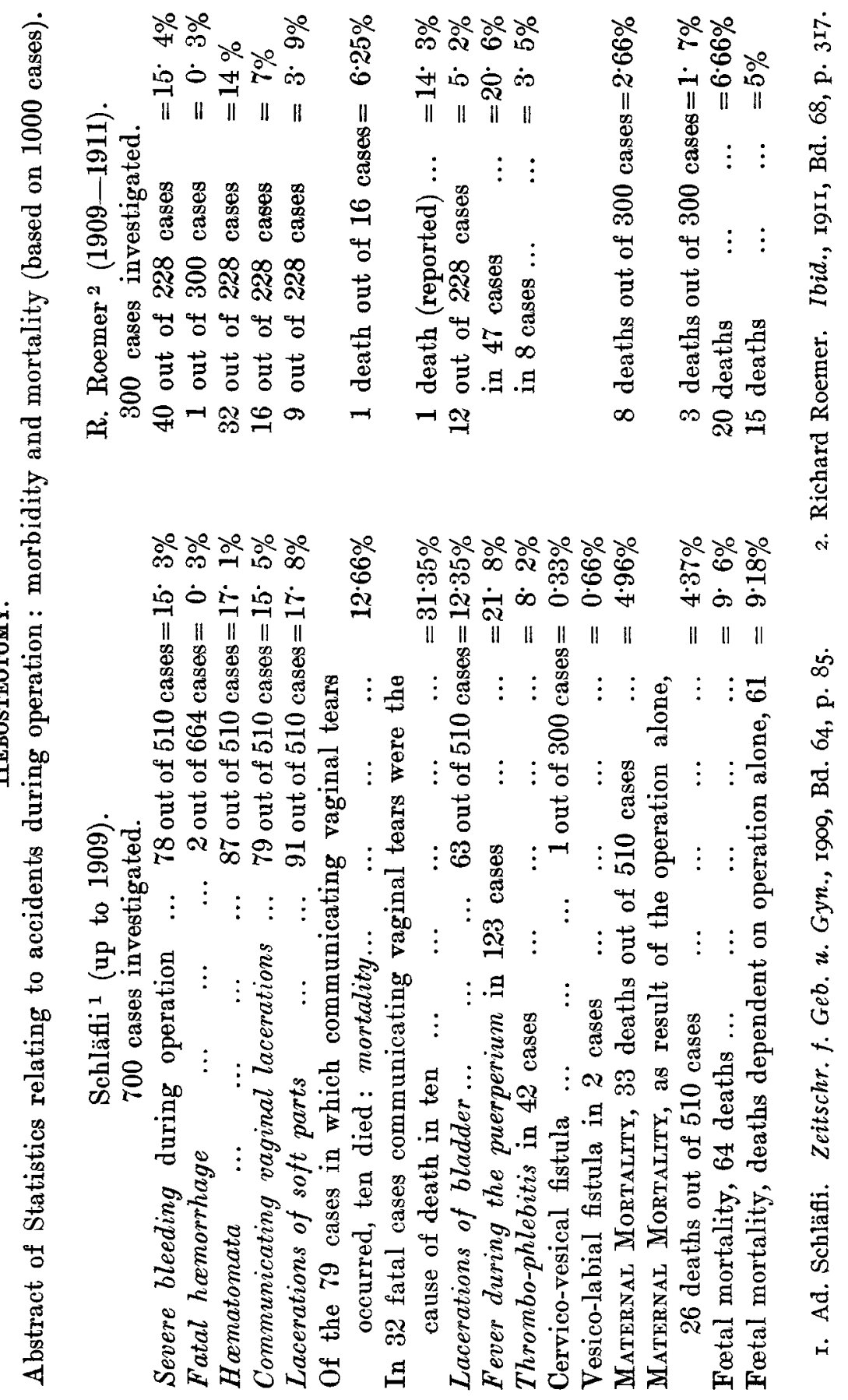


Other sequelæ, too numerous for mention, have been recorded, but there may be noted:-infections of the cellular tissues of the pelvis, infections of bone with sequestrum formation, and in the case of the open operation, hernia through the divided sheath of the rectus muscle.

Were they universal, results so deplorable would require the abandonment of the operation, but fortunately a few obstetricians have not assumed it to be a panacea for every form of pelvic contraction, for instance one of the pioneers, van de Velde, who recently recorded the 14 cases which have taken him ten years to compile, with good results in every instance. The truth is that hebosteotomy has been too widely regarded not only as a competitor with induction of labour, with the high forceps operation and craniotomy, but also with Cæsarean section, and it has been applied in scores of cases for which it was entirely unsuitable.

Cæsarean section eludes mechanical difficulties of all degrees: the aim of hebosteotomy should be to overcome those slighter varieties which are a menace to the life of the child. If mechanical difficulties are encountered after division of bone, then the performance of the operation must be set down as an error of judgment, for success is only obtained when delivery is easily effected, without compression of the foetal head, without laceration of the mother's soft tissues and without undue distortion of her sacro-iliac joints. A tight fit of the head in the pelvis implies all the risks of the high forceps operation plus injury to the pelvic joints and infection of the severed bone.

The operation has therefore very narrow limits, and it cannot be one of frequent performance. When reporting my two cases in 1906 I stated my belief that it was called for only in those cases in which forcible extraction by forceps promised to result in the delivery of a dead child, but in which the addition of an extra centimetre to the conjugate seemed to give a reasonable chance of delivering a living one. To this opinion I still adhere. It is usually stated that for each centimetre of gap between the severed ends of bone, the $\mathrm{Cv}$. is increased $2 \mathrm{~mm}$., so that a separation of two inches would give the extra centimetre required (2/5 inches). In my limited experience such a separation can be safely effected. It must not be forgotten that all the pelvic diameters are enlarged and that there is consequently a marked increase in the cubic capacity of the pelvis.

In cases of the kind mentioned, where the patient is free from suspicion of infection, and the head lies lowest in the uterus, hebosteotomy will probably survive. But it can never be regarded as a substitute for what Leopold terms "that most elegant of operations "-Cæsarean section. 